\title{
Roundabout Situational Awareness for Automated Vehicles with Hybrid Machine Learning Approach
}

\author{
Mehran Zamani Abnili' ${ }^{1}$ Nasser L. Azad ${ }^{2}$ \\ 1,2 University of Waterloo \\ 200 University Ave. W., Waterloo, Canada \\ mzamania@uwaterloo.ca; nlashgar@uwaterloo.ca
}

\begin{abstract}
In this paper, a hybrid approach for situational awareness in roundabouts is presented that can produce traffic participants' behaviour for arbitrary horizons. This real-time implementable strategy consists of dynamic Bayesian network and a continuous variable prediction module (CVPM) as its subparts, making it a data-driven approach while providing the facility to incorporate experts' knowledge into the predictions. Being a data-driven approach, the data is obtained using SUMO as a simulation platform, and three different CVPMs are experimented with, namely recurrent neural network (RNN), gated recurrent unit (GRU), and long short-term memory networks (LSTM). The chosen RNN yields a correlation higher than 0.895 and RMSE less than 0.036 for 10 seconds predictions.
\end{abstract}

Keywords: Machine learning, situational awareness, traffic participants behaviour prediction, dynamic Bayesian network, recurrent neural network, gated recurrent unit, long short-term memory

\section{Introduction}

Automated driving has been an area of interest for both researchers and industries for a long time but lagged behind in automation due to the complexity of the problem. Where in other industries the automation can replace the conventional manual labour often instantaneously, in driving that is not an option as vehicles are privately owned and operated. This results in an extended transition period where potential autonomous vehicles are supposed to operate alongside their humandriven counterparts. This adds a lot of complexity to the problem as human erratic behaviour translates to a chaotic environment. Moreover, the infrastructure where vehicles are deployed in is also not designed for automated vehicles but is tailored for a human operator. As a result, not only controlling an automated vehicle is a challenge, but also for that vehicle to understand its surroundings and being able to interpret its situation is not a trivial task. Hence, the indispensable need for a perception strategy becomes apparent.

The motivation behind the drive pushing the autonomous vehicles research is quite simple and has 4 main aspects, aside the obvious industry race toward being the first producer of fully autonomous vehicles for personal gains. The four main aspects can be enumerated as:

1- Affordable long-range transportation for the public.

2- Personal transportation for individuals with disabilities.

3- Diminishing of traffic casualties associated with human error.

4- Minimization of harmful exhaust emissions. [1]

Putting that beside the fact that most of traffic accidents are solely caused by human errors rather than hardware failures and other occurrences, and out of those caused by human errors, most being due to recognition errors [2, 3] it reinforces the need for a perception strategy.

The research in this area has seen a lot of interest in the past decade mostly due to the new and improved computer hardware, making machine learning algorithms plausible at all, as conventional control algorithms are not able to deal with the difficulties in a satisfactory way. However, the readers who are not themselves working in this area should understand that with all the myriad endeavours that has gone into this subject, there is still much to be discovered and many solutions to be found for the vastness and complexity of autonomous driving problem.

The contribution of this study is in the presentation of a hybrid machine learning situational awareness approach for roundabout driving, which is an extension of the work reported in [4]. This approach can incorporate an expert's knowledge (e.g. traffic laws) into traffic behaviour predictions for any variable and arbitrary horizons using a combination of a dynamic 
Bayesian network (DBN) and a continuous variable prediction module (CVPM) that will be discussed in more detail in the upcoming sections. The strategy is applied to the case of roundabout driving which has been experiencing an emergence especially in growing urban areas as roundabouts offer a passive solution to traffic control which in turn makes them 'smarter' in controlling traffic. The lack of active components, for instance a traffic light signal, at roundabouts means that they are adaptive to the flow and temporal changes in traffic, but in turn poses a threat to inexperienced drivers who are not acquainted with roundabout driving, often causing a chain reaction in traffic accidents.

Compared to other relevant studies where a narrow scope of autonomous driving is discussed or tackled, this approach is very versatile and can be adapted to virtually any driving scenario. As an example, in the study conducted by Zhang et al. [5], while the approach is able to make predictions on a single drive cycle, it does not consider context, presence of multiple traffic participants, or even lateral motions, and only focuses on a longitudinal motion of a carfollowing model. Similarly, in the study conducted by Sun et al. [6] while multiple CVPMs are compared, the approach does not consider multiple variables and extension of the same approach for multiple variables will not encompass the dependencies between those variables. Similarly, Thorsell's [7] approach can make single drive cycle predictions without considering spatial information. There are many other studies following the same principles, and that is what makes this study unique. This approach considers context, can adapt to the situation, and the predictions can be done on any variable while their dependencies are conserved. However, for the sake of ease of comparison, this study also focuses on velocity predictions. The following sections will go over the basics and the background of the approach, then the strategy is discussed, and finally results are declared and discussed, followed by conclusions and future work.

\section{Background}

Disclosing the innerworkings of all the components that go into making the predictions possible would be outside the scope of this paper, but to understand the work, some background should be declared. There are two main components in the prediction strategy which will be discussed in the following subsections, but these two components are a DBN and a CVPM as mentioned above. General schematics of these two components and how they are connected is illustrated in Fig. 1.

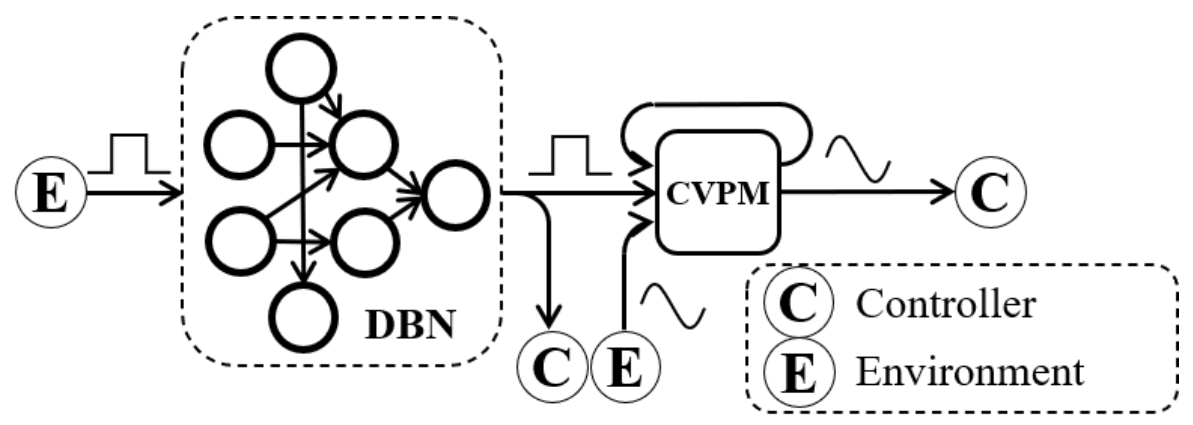

Fig. 1 Overview of Method

In Fig.1, the case of controller is a hypothetical one and as it will be discussed in the conclusion, this prediction strategy is a prime target to be used with model predictive controllers (MPCs) to provide them with accurate state predictions. The other main components are the DBN, and the CVPM.

\subsection{Dynamic Bayesian Network}

A Bayesian network [8] is a graphical representation of probabilistic variables where the connections serve as dependencies between those variables. In a driving context, most of the variables can be categorized as probabilistic and their values can be classified into mixture distributions. As an example, a traffic light signal has 3 states (4 if considering advanced left). Similarly, 'current lane of driving' has a limited number of states. Even in some cases, where the actual value of a continuous variable is not critical, continuous variables can be turned into a mixture distribution by introducing semantic rules. For instance, for a variable such as distance, anything past a threshold can be dubbed "far" and below 
that threshold "near". As far as mixture distributions and discrete variables are concerned, DBN is a powerful tool that can obtain likelihood of each variable taking a specific state given a set of conditions. As mentioned in the introduction, the purpose of the perception middleware is to be able to understand traffic participants behaviour and incorporate expert knowledge into understanding the environment. This approach is replicating the learning process of driving for a human. A A human driver is not adequately equipped to be able to measure distances and speeds numerically but will always know, know, semantically, whether an object is too far or near, or too fast or slow. Also, a human driver by knowledge and experience knows that the lane in which a vehicle enters an intersection in will govern its destination. There is always a degree of uncertainty, but the human driver can adapt their initial assumption if given a set of observations.

Bayesian networks can be classified as expert systems in the sense that the topology can be defined by an expert which will provide the network with an initial understanding of the relationship between variables. They are also a data-driven approach that can learn from large datasets quickly. The dataset will be stored as sets of Gaussian normal distributions of $\mathrm{P}(\mathrm{A} \mid \mathrm{Pa}(\mathrm{A}))$, where $\mathrm{A}$ is a probabilistic variable and $\mathrm{Pa}(\mathrm{A})$ is the set of its parents (i.e. set of variables with an edge toward A). Once fully learned, any joint or conditional probability between variables can be computed. The learning process in Bayesian networks is done through EM algorithm, a descendent of the forward-backward algorithm. In this algorithm a set of Gaussian normal distributions are fitted to mixture distributions by generating $\mathrm{n}$ random Gaussian distributions, finding the likelihood of each sample belonging to each distribution and then updating the distribution properties based on classed samples. [9] DBNs are Bayesian networks with temporal nodes which are variables with values from the next time-slice in the training. However, not every variable in a driving context can be represented as a mixture distribution. Variables such as speed are continuous, and their value is often desired. DBN is not adequately equipped to make predictions for continuous variables, hence there is the need for a CVPM.

\subsection{Continuous Variable Prediction Module}

The continuous variable prediction module can be any data-driven sequence prediction method. In machine learning realm, primitively a feed-forward neural network can be equipped with a feedback loop and unit delays to become a recurrent neural network (RNN). An RNN can then be trained to take a short history of the variable as the input and produce predictions for the next time slice. By repeating the cycle, assuming the predicted value as the true current value and cascading the history one step back, each the prediction can be extended to an arbitrary horizon. Although it is common knowledge that as the horizon becomes larger the accuracy suffers because of the compounding effect of the errors.

RNNs are known to struggle with the vanishing gradient problem. The vanishing gradient problem is, in essence the stoppage of training due to the shrinking error gradient in the back-propagation algorithm. The opposite of gradient explosion problem is where the large gradient will cause the training to diverge from the global optimum. A solution to this problem is using newer methods such as the gated recurrent units (GRU) or long short-term memory networks (LSTM) training with stochastic gradient descent (SGD), ADAM or other algorithms. Although the workings of these algorithms are outside the scope of this paper, in this study we have compared the performance of some of these methods which will be discussed in more detail in the results section.

\section{Implementation strategy and results}

As mentioned in the previous section, the approach taken in this study is a data-driven one. The first step toward developing a data-driven prediction strategy is to understand the dataset and know what variables are available to work with. As available datasets are often lacking in useful data as data collection is generally time consuming and difficult to the point where it is completely implausible, the refuge is to simulation. In this study, a simulation environment was implemented in SUMO [10]. To clarify, the only difference between using real data and simulation for the case of making predictions is extra pre, and post processing steps which are trivial to the core of the prediction methodology. However, it is important to note, the previous statement only applies to law-abiding norm. If the networks are not trained for car crashes, or aggressive manoeuvres, they are not able to predict such occurrences. 


\subsection{Simulation in SUMO}

The scope of this study is roundabout driving which is emerging in developing cities. SUMO does not support roundabouts natively, so the environment was created using a number of road sections connected via zippers in a circular shape. The increased number or zippers also acts as a lane-change preventing solution for inside the roundabout. In the considered roundabout, the priority for travellers has been increased so that the simulation is as close to reality as Each arm has extended for 500 meters as two lanes with a different traffic flow density to introduce variations into the A total of 16 flows were introduced at the end of every arm, one introducing one vehicle at every time step on the west side, one introducing one vehicle at every 2 time steps on the south side, one introducing one vehicle at every 3 time steps on the north side and one introducing one vehicle at every 4 time steps on the east side. In other words, the opposing sides have an equal total flow but placing the observer at each side would produce variations in the data. The flows have combined random trip destinations so that from every arm the flows generated will travel every four arms, but the sequence in which each vehicle is spawned into the simulation is random. A snapshot of the simulation environment is illustrated in Fig. 2.

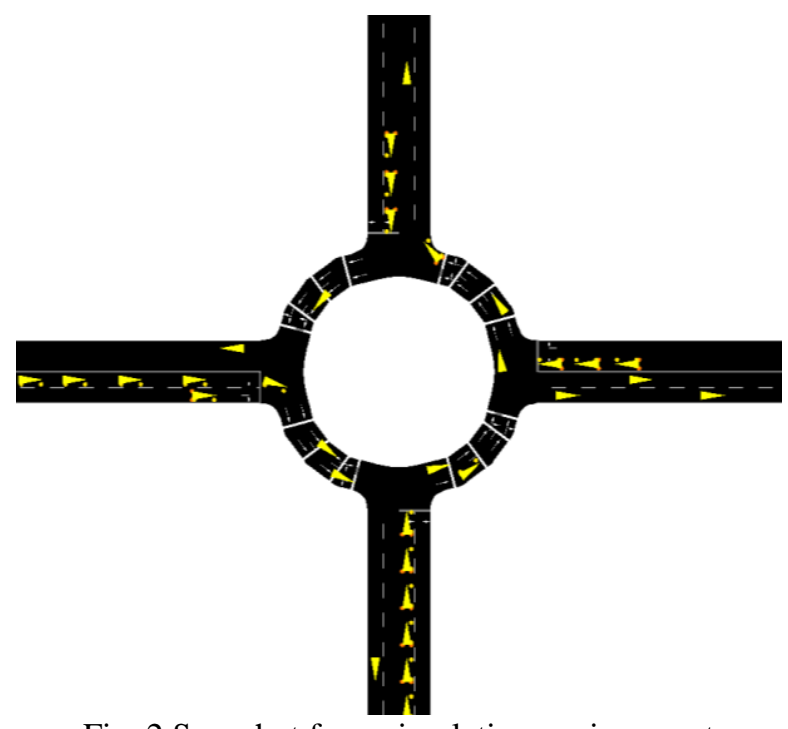

Fig. 2 Snapshot from simulation environment

\subsection{Dynamic Bayesian Network Topology and Variables}

The data extracted from SUMO has information about positions, speeds, and lanes of the 3000 vehicles in the simulation. The position information can be fused with the map of the environment to obtain relative positions of vehicles conserving the traffic rules. Due to the extreme size of the dataset, spectral clustering was performed on the set of vehicle pairs that were co-present in the simulation. In a $3000 \times 3000$ sparce grid of cells, the cells with data were rearranged to the closest configuration to a diagonal matrix. This rearrangement of data allows for much shorter processing times when extracting data for the DBN. The DBN topology is not guaranteed to be optimal and relies on the experts' subjective judgement. In this case, the variables were chosen with two main objectives in mind:

1. Having the least pre-processing requirements.

2. Being the most convenient to measure from the environment in a real driving scenario.

After the spectral clustering, the following variables were extracted from the dataset by fusing the position, speed, and lane data with the roundabout layout. The variables, their states, and their layout can be found under Table 1. 
Table 1: DBN Variables, their states, and their layout

\begin{tabular}{|l|l|l|}
\hline$\#$ & Variable Name & Variable States \\
\hline 1 & Lane & $\{1,2\}$ \\
\hline 2 & Start Position & $\{\mathrm{E}, \mathrm{N}, \mathrm{W}, \mathrm{S}\}$ \\
\hline 3 & Entered $\mathrm{RA}^{1}$ & $\{$ True,False $\}$ \\
\hline 4 & Lane in RA & $\{1,2\}$ \\
\hline 5 & Destination & $\{\mathrm{E}, \mathrm{N}, \mathrm{W}, \mathrm{S}\}$ \\
\hline 6 & Inside RA & $\{$ True,False $\}$ \\
\hline 7 & Stop to Enter & $\{$ True,False $\}$ \\
\hline 8 & Lane Prime & $\{1,2\}$ \\
\hline 9 & AccFlag & $\{$ Negative, non-Negative $\}$ \\
\hline 10 & AccFlag Prime & $\{$ Negative, non-Negative $\}$ \\
\hline
\end{tabular}

\subsection{CVPM Implementation and DBN Interfacing}

This study experiments with a variety of CVPMs to find the best one for this application. The most common sequence predictors used in this art are the vanilla RNN, GRUs and LSTMs, therefore those are the methods of choice for this study. The input-output for the three different implementations are the same and consist of a 12-second velocity history, complemented by the DBN stream. The DBN stream consists of the likelihood of one setting for binary variables, and the mean and the likelihood of the setting with maximum likelihood for non-binary variables. In this specific application, due to low number of DBN nodes, it was possible to feed all the variables into the CVPM to complement the velocity history. To keep it fair between the three methods, the number of layers and nodes were kept consistent throughout the trials, as well as training properties. One thing to keep in mind, however, is that in the case of complementary inputs, the DBN stream in this case, the closer the sequence predictor structure to a perceptron, the smoother the output and the higher the accuracy. This is validated by the results illustrated in the following figures. Also to keep the results fair, Kalman smoothing was not implemented in these results, however, Kalman filter has shown to improve the prediction results in [4].

Stacked plot with the RNN, GRU, and the LSTM results are generated and presented in Fig. 3 with the errors illustrated in Fig. 4. The results for the RNN are far superior than that of the GRU and LSTM in this specific application and that is beside quicker training times and turnaround times. Also, in this specific application, between the GRU and the LSTM, the LSTM has the upper hand in terms of accuracy.

As the vanilla RNN proved to be the most accurate among the methods, we focused on the RNN as the method of choice. Fig. 3 illustrates the prediction results for a vehicle that spent a relatively short period in the simulation. The low average speed and frequent stops are markers that there was significant amount of traffic present during this trip, however the traffic has not caused the traffic to stop completely. That is as opposed to the trip taken in Fig. 5 where the trip takes three times longer for the same distance travelled. Either result demonstrates very high prediction accuracy for both low speed traffic driving and following the transients. The complementary DBN stream has the effect on velocity prediction where there is clear distinction between low speed travel and complete stop, even for extended periods. The way DBN can achieve this is introducing a switch with many configurations each having a different weight that the RNN learns through back propagation algorithm. The error bounds for normal driving scenario for the RNN are [-0.71,0.94], [-0.97,1.47], [-1.46,2.75], and [-

\footnotetext{
${ }^{1}$ RA stands for "Roundabout"

2 "Prime" refers to the variable in the next time-slice

${ }^{3}$ Acceleration Flag
} 
2.11,4.10], and in the case of heavy traffic driving are also measured at $[-0.75,1.23],[-1.58,2.25],[-4.62,4.63]$, and [$6.64,6.74] \mathrm{m} / \mathrm{s}$ for $1,2,5$, and 10 second predictions respectively. One notable item in these results is that low speed predictions are a lot more accurate than the final high-speed transients as the vehicle is jetting off the simulation
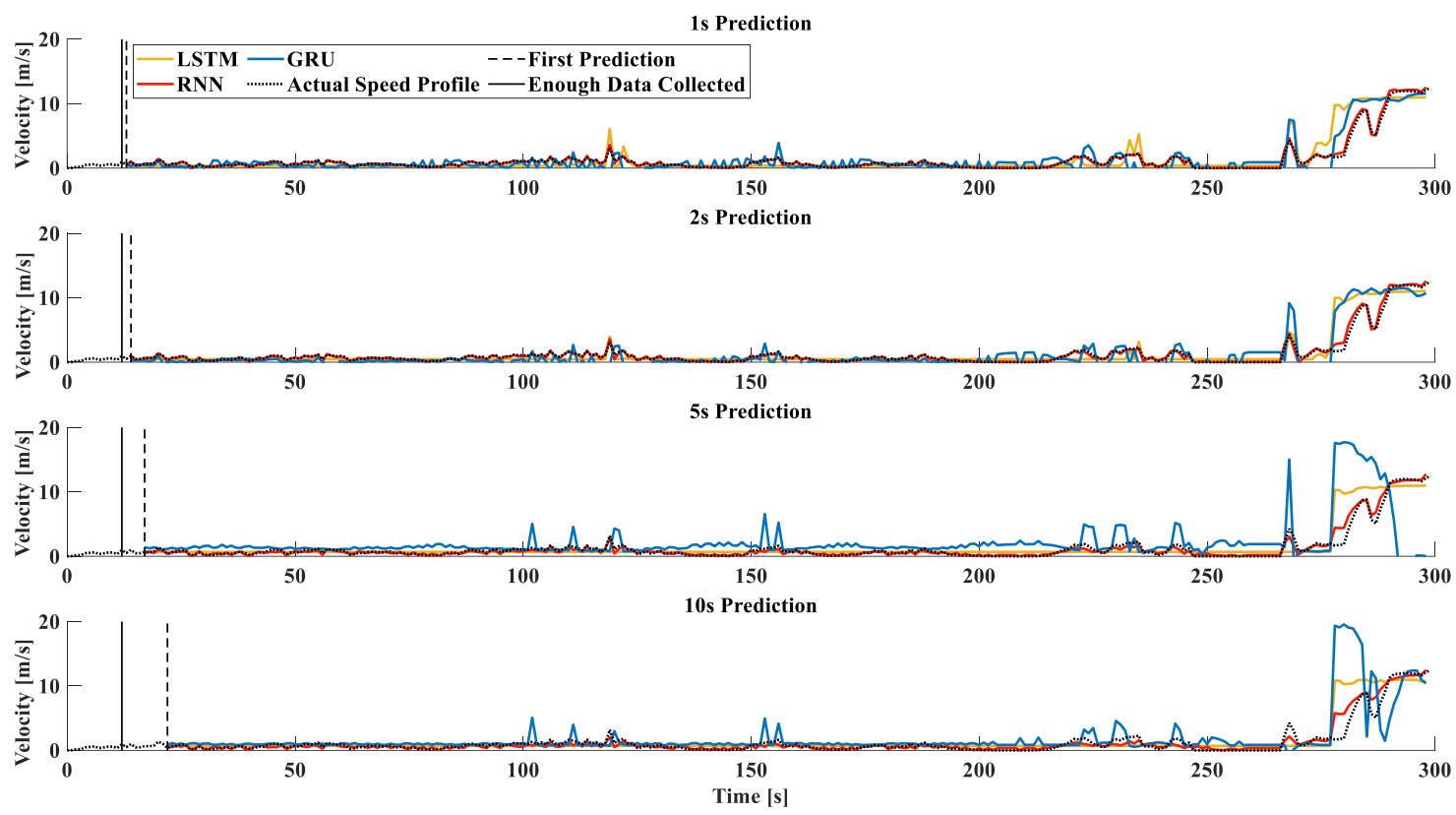

Fig. 3 DBN-RNN results for normal roundabout driving scenario
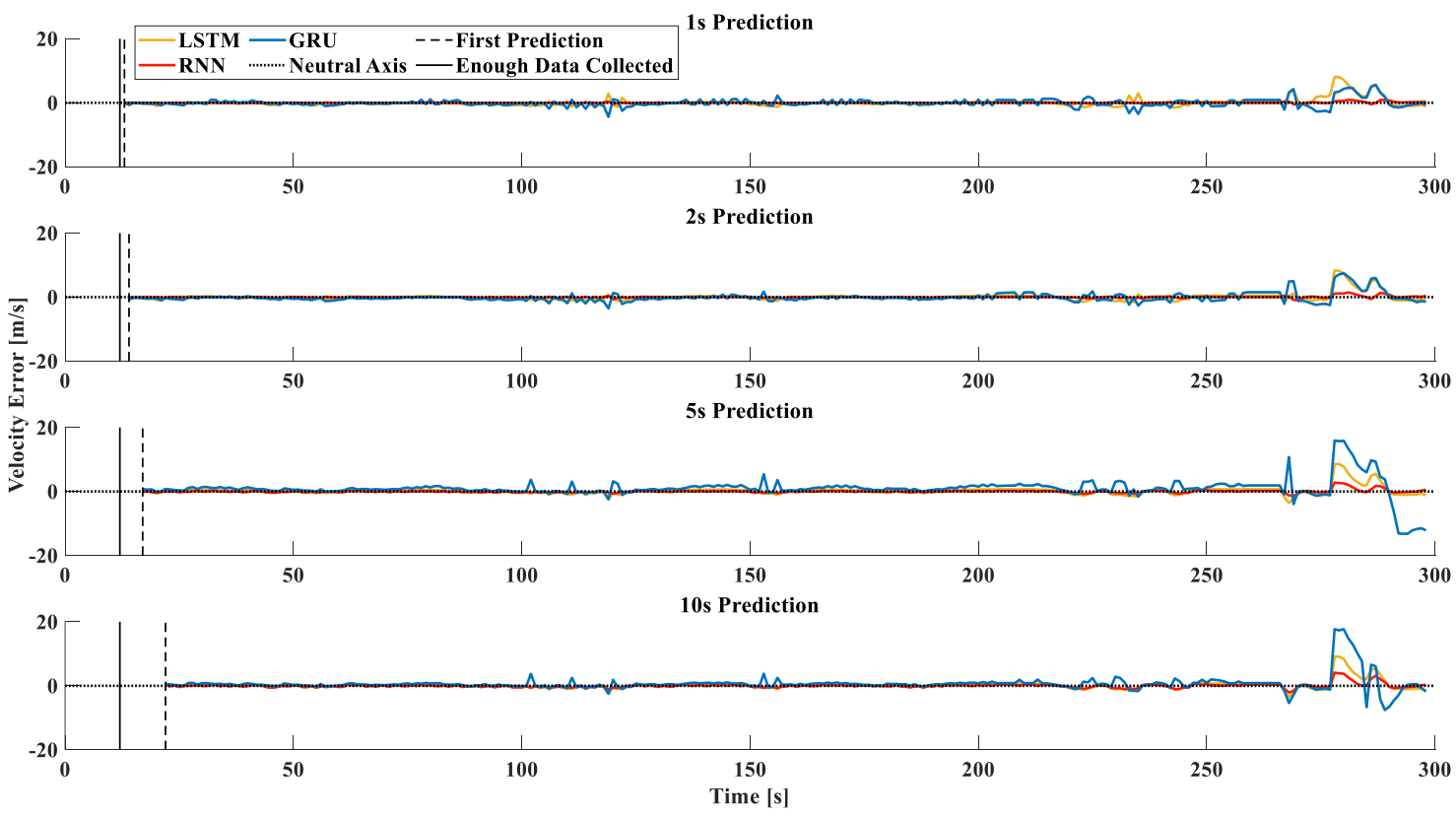

Fig. 4 DBN-RNN Errors for normal roundabout driving scenario 

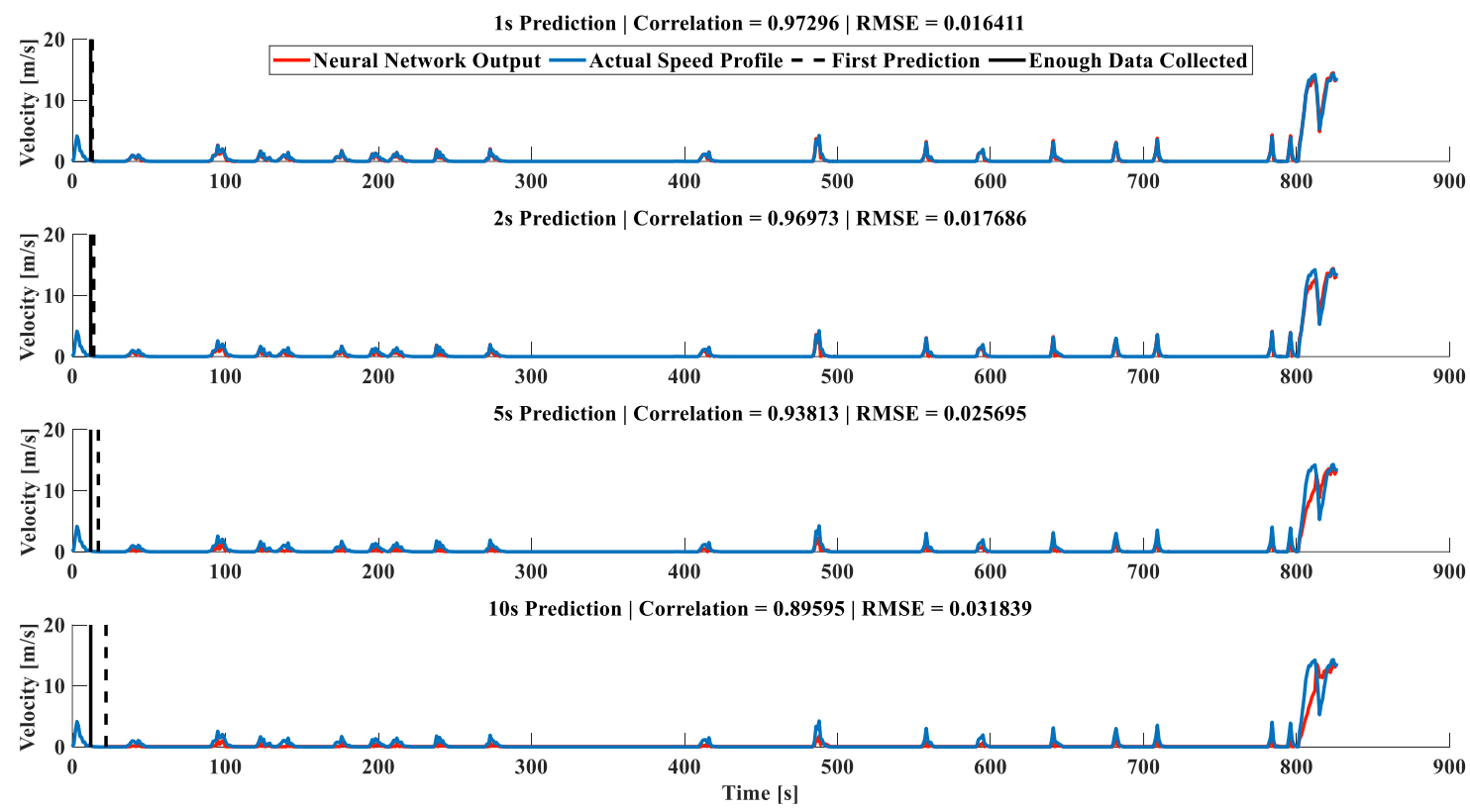

Fig. 5 DBN-RNN results for heavy traffic

The reason for this phenomenon can be explained by analysing the dataset. In this dataset, vehicles that spent longer inside the simulation (due to heavier traffic), introduce more samples in the training. Out of these 'extra' samples most, if not all of them, are zeros or very small values. That is because the travelled length is equal for all vehicles and that would be the area underneath the speed curve. The only scenario where these 'extra' samples are not zero are for the cases where there is one or more lane-changes going into, or out of the roundabout. There are ways to mitigate this issue by introducing low traffic periods and merging multiple datasets for different flow densities. However, as some readers may have noticed, the main objective of a roundabout prediction strategy is to make predictions for a merging vehicle into the roundabout, and that is where the challenge lies, as vehicles inside the roundabout have higher priority and can exit anytime without hesitation. In other words, once a vehicle has merged into the roundabout the problem is solved for the most part, and to predict a vehicles motion after the roundabout, much simpler solutions can be implemented like the one introduced in [11].

Finally, the accuracy and validity of the results presented were measured by cross-correlations between actual data and the predicted speed profiles. These metrics for the RNN in the scenario presented in Fig. 3 are shown in Table 2. As expected, the higher the prediction horizon, the lower the accuracy, but the results maintain a correlation greater than 0.895 and an RMSE less than 0.036 .

Table 2: Accuracy metrics for RNN in scenario presented in Fig 4.

\begin{tabular}{|l|l|l|}
\hline Prediction horizon & Correlation & RMSE \\
\hline 1 second & 0.97641 & 0.029334 \\
\hline 2 seconds & 0.97792 & 0.028453 \\
\hline 5 seconds & 0.97661 & 0.029249 \\
\hline 10 seconds & 0.96462 & 0.035995 \\
\hline
\end{tabular}

\section{Conclusion and Future Work}

This study proposes a versatile, real-time implementable traffic participants behaviour predictor and situational awareness strategy for roundabout driving. The results of this study can be integrated with model predictive controllers 
similar to the ones in [12] and [13] to improve performance and achieve autonomy in specific driving scenarios where expert data is available, and the general layout of the environment is known. Although there is no guarantee that the expert-yielded topology for the DBN is the optimal one, the one proposed in this specific use-case proved to be accurate and produced valid results for different scenarios, however, to achieve a well-rounded package that can produce accurate predictions for any roundabout, two aspects need to be improved. The hypothetical situational awareness package needs to be trained with data considering all infrastructure layouts, and also needs to be able to identify the situation before generating the table of variables and their states, similar to the one in Table 1. However, that would be an extension of this very method and the principles remain the same.

\section{Acknowledgements}

The authors would like to thank Toyota, NSERC, and OCE for their generous support of this project.

\section{References}

[1] D. Watzenig and M. Horn, Automated Driving Safer and More Efficient Future Driving, Springer International Publishing, 2018.

[2] 2015 Motor Vehicle Crashes: Overview, NHTSA's National Center for Statistics and Analysis, 2016.

[3] Critical Reasons for Crashes Investigated in the National Motor Vehicle Crash Causation Survey, 2015.

[4] M. Zamani Abnili and N. L. Azad, "Short-Term Traffic Participants Behavior Prediction Using A Novel Hybrid Method in Highway Merging for Automated Vehicles," IET Intelligent Transportation Systems (Under Review).

[5] F. Zhang, J. Xi and R. Langari, "Real-Time Energy Management Strategy Based on Velocity Forecasts Using V2V and V2I Communications," IEEE Transactions on Intelligent Transportation Systems, vol. 18, p. 416-430, 2017.

[6] C. Sun, X. Hu, S. J. Moura and F. Sun, "Velocity Predictors for Predictive Energy Management in Hybrid Electric Vehicles," IEEE Transactions on Control Systems Technology, vol. 23, p. 1197-1204, 2015.

[7] E. Thorsell, "Vehicle speed-profile prediction without spatial information," 2013.

[8] J. Pearl, Bayesian networks, Computer Science Dept., University of California, 1998.

[9] Z. Ghahramani, "Learning dynamic Bayesian networks," in International School on Neural Networks, Initiated by IIASS and EMFCSC, 1997.

[10] P. A. Lopez, M. Behrisch, L. Bieker-Walz, J. Erdmann, Y.-P. Flötteröd, R. Hilbrich, L. Lücken, J. Rummel, P. Wagner and E. Wießner, "Microscopic Traffic Simulation using SUMO," in The 21st IEEE International Conference on Intelligent Transportation Systems, 2018.

[11] M. Zamani Abnili and N. L. Azad, "Short Term Predictions of Preceding Vehicle Speeds for Connected and Automated Vehicles," Controls, Dynamic Systems and Robotics (CDSR), 2019.

[12] M. Vajedi and N. L. Azad, "Ecological Adaptive Cruise Controller for Plug-In Hybrid Electric Vehicles Using Nonlinear Model Predictive Control," IEEE Transactions on Intelligent Transportation Systems, vol. 17, p. 113-122, 12016.

[13] S. Tajeddin, S. Ekhtiari, M. Faieghi and N. L. Azad, "Ecological Adaptive Cruise Control With Optimal Lane Selection in Connected Vehicle Environments," IEEE Transactions on Intelligent Transportation Systems, p. 1-12, 2019. 